\title{
Commentary \\ The Role of Support Groups, Advocacy Groups, and Other Interested Parties in Improving the Care of Patients with Congenital Adrenal Hyperplasia: Pleas and Warnings
}

\author{
Peter A. Lee ${ }^{1,2}$ and Christopher P. Houk ${ }^{3}$ \\ ${ }^{1}$ Department of Pediatrics, Penn State College of Medicine, The Milton S. Hershey Medical Center, P.O. Box 850, \\ 500 University Drive, Hershey, PA 17033-0850, USA \\ ${ }^{2}$ Section of Pediatric Endocrinology, Riley Hospital for Children, Indiana University School of Medicine, Indianapolis, \\ IN 46202, USA \\ ${ }^{3}$ Department of Pediatrics, Medical College of Georgia, Augusta, GA 30912, USA
}

Correspondence should be addressed to Peter A. Lee, leepa@iupui.edu

Received 1 April 2010; Accepted 27 April 2010

Copyright (C) 2010 P. A. Lee and C. P. Houk. This is an open access article distributed under the Creative Commons Attribution License, which permits unrestricted use, distribution, and reproduction in any medium, provided the original work is properly cited.

\begin{abstract}
In the era of advocacy groups, it seems appropriate to contemplate how best to utilize them for patient benefit in the management of those with disorders of sex development (DSD), including those with congenital adrenal hyperplasia (CAH). Such interactions, to be constructive, require a spirit of cooperation to optimize outcomes. A traditional view of advocacy groups as a type of defender of patients' rights appears outdated and it is time that the benefits of their participation be fully realized. Open dialogue with all patients/families, including those who feel harmed by prior care are paramount. We discuss several recent examples of interactions that illustrate how dialogue in the name of "advocacy" can have a negative impact on developing a framework for ongoing constructive dialogue and actions. Such approaches completely change the dynamics of subsequent interactions. Physicians involved in the care of individuals with DSD, including those with CAH, and patients should be aware of confrontational techniques and legal implications that may be used by some advocacy groups. Hopefully recent efforts to promote a multidisciplinary care approach for patients with DSD/CAH will continue to foster mutual cooperation between team members, where the common goal is improving patient/family outcomes and quality of life.
\end{abstract}

\section{Background}

It may be appropriate to review the objective of Rotary International- to encourage and foster the ideal of service as a basis of worthy enterprise [1]. This involves the development of opportunities for service, maintenance of high ethical standards in business and professions, the recognition of the worthiness of all useful occupations, and the dignifying of every occupation as an opportunity to serve society. Their philosophy is expressed in the "Rotarian four-way test" [2], a type of litmus test used to determine whether a planned action is compatible with the Rotarian spirit. The questions posed by the "four-way test" are the following (1) Is it the truth? (2) Is it fair to all concerned? (3) Will it build goodwill and better friendships? and (4) Will it be beneficial to all concerned? The authors submit that such a test would make a good standard for assessing the actions of support and advocacy groups involved in obtaining and sustaining the best care for patients with congenital adrenal hyperplasia (CAH) and other disorders of sex development (DSD).

In recent decades, there have been many positive changes toward a more humanistic approach to medical care provided to patients [3-5]. This has certainly been true for the person born with $\mathrm{CAH}$, with or without ambiguous genitalia. This is likewise true for other DSDs. Basic among these changes are full disclosure of all known medical information and findings to the patient and their families, respect for patient privacy with regard to unnecessary physical exposure during clinical examinations for teaching purposes, and the inherent right of parents or patients to make decisions regarding therapy. These include in some instances gender assignment, after being fully informed of 
the medical and psychological ramifications. We strongly believe all of these changes pass the four-way Rotarian test. The community of health care providers has progressively adopted these approaches.

Optimal care involves a group of well-informed, wellintentioned, and experienced team members whose primary goals are to provide all pertinent aspects of medical information, the potential therapies, and the advantages and disadvantages of each. To accomplish this, a team effort with all participants focusing on the common good of the patient(s) with a basic respect for other team members' differing opinions and perspectives is imperative. Support groups have aided considerably in this effort during recent years, with a key role for them to advise and counsel newly diagnosed patients or, in the situation of the newborn, parents of the neonate. This has at times developed into a support group representing older patients about their concerns with their previous medical care. Support groups such as the MAGIC (Major Aspects of Growth in Children) Foundation and the CARES (Congenital Adrenal Hyperplasia Research Education \& Support) Foundation have provided such needed advice and support, as did the Intersex Society of North America (ISNA).

Whenever situations involve a change in the standard of care, as has occurred for those with DSDs, and whenever there are instances involving strong emotions, there is a high risk of polarization. All involved must be alert to this and work to manage and mitigate these risks and biases. It is tempting to conclude that the potential risk for polarization is high for support/advocacy groups. These groups are likely to have a primarily emotional approach to the problem. When combined with a high degree of zeal to help plus defensive reactions of interactions with others involved in helping patients with DSDs, the outcome is less likely to be constructive. These groups also risk becoming so focused on one particular aspect of care that they lose sight of the value of long-term experience or in-depth education about the multiple ramifications of complex medical conditions.

\section{Perspectives}

This article is written from the perspective that collaboration is the most productive approach and confrontation, coercion and intimidation are likely to be divisive and, in fact, may undermine any beneficial patient outcome. Confrontational tactics used by "advocacy" groups have included pressuring the medical community to adopt narrow mandates, such as a moratorium on all reproductive system surgery [6], that cannot apply to the broad range of situations encountered in practice or the use of accusations regarding therapy received by patients in the past. These tactics are currently being employed by well-meaning individuals whose passion for confrontation seems to cloud the recognition that multiple factors are involved in patient outcomes, many of which are beyond the control of the healthcare team. These individuals may also fail to recognize that prior care was within the standard of care that existed at the time. Much of this type of misinterpretation of information and incomplete knowledge of the topic is a common part of human communications.
Further, as has been noted in other situations of early childhood (and illustrated below), the patients may not actually remember the details $[7,8]$, the timing, or the sequence of decisions, may confuse personal experiences with those of others, and may simply lack the knowledge or understanding for the reasons for the treatment decisions that affected them. Such recall bias may lead to chronic resentment, which ultimately can impede lifelong adjustment.

With the recent proliferation of advocacy and support groups, there are examples of confrontational methods in attempts to rectify treatments. These are exemplified by: (1) publication by CARES Foundation [9] of a publication of the Endocrine Society's Research Affairs core committee [10] which reviewed the pros and cons of the prenatal therapy of at risk fetuses including the conclusion which called for abandonment of prenatal treatment of $\mathrm{CAH}$; this together with (2) a group of bioethicists objecting to the use of prenatal dexamethasone therapy given to pregnant women at risk of having a $46, \mathrm{XX}$ fetus with $\mathrm{CAH}$, a practice that may have quickly become too common, illustrating one of numerous therapies presumed, but not proven, to be safe [11], that has been adopted without clear FDA approval for the specific indication, exemplified by the cited example [12]; (3) Advocates for Informed Choice, which on one hand very appropriately promotes giving pediatric patients a voice in treatment of DSD $[13,14]$, while on the other has demanded apologies from institutions and physicians who cared for children with DSD in the past and regularly publisher articles in a nonpeer reviewed, free periodical. These publications include the statement that gonadectomy in an infant with DSD is comparable to castrating a mentally retarded child to preclude fertility [15]. Unfortunately, this style to draw attention often does not lead to an informed identification of a problem and can be particularly destructive to the development of a balanced resolution. Conversely, if parties from both sides of this issue could have open discussions, an informed viewpoint that would benefit all involved (Rotarian rule no. 4) can be promoted.

Such approaches are fraught with the possibility of misrepresentation and are at risk to result in more harm than good. The failure to fully explore all aspects of a complex issue or the alienation of those with years of experience caring for children with DSDs may be detrimental. In addition, these types of complaints are typically not based on a representative survey of patient outcome data. They risk the possibility that some may aggressively defend the dissatisfied person without a full knowledge of the uniqueness of that individual.

An additional problematic aspect of several support groups is that gay, lesbian, or gender dysphoria has been added to the list of patients represented. Trying to lump together multiple types of patients merely based on the assumption that all are dissatisfied creates the risk of advocating against therapies that may be uniquely beneficial for patients with DSDs. While there are aspects where children with DSDs, transsexual, gay, and lesbian persons overlap, there are many other areas in which the issues are substantially different. There is a danger that gender dysfunction aspects will be overly emphasized if DSDs are 
approached like physically normal people with sexual orientation and transsexual concerns. On one hand, those have gender issues but having been born with fully differentiated genitalia can relate very well with those who were born with genital ambiguity; on the other, a whole spectrum of other factors may be involved for the person with a DSD such as the need for surgery, fertility issues, the effect of having "different" genitalia, or the need for chronic medical management.

To be constructive, support groups should begin to view the controversial issues in DSD management from broader perspectives that can be focused on areas of need. These include approaching (1) parents at the birth of a child with CAH or DSD, (2) approaching the child and parent as the child matures and becomes able to understandhis $\backslash$ her condition and to assume responsibility for decisions regardinghis $\backslash$ her care, and (3) reviewing the ongoing needs of adult patients who may feel that previous standard of care treatments were done with a malicious or callous intent.

\section{Recall Fallacies}

It is known that recall of specifics from early childhood traumatic situations may be inaccurate $[7,8]$. By citing information below from three patients born with genital ambiguity, two of whom have $\mathrm{CAH}$, it is shown that the individuals' knowledge of decisions and disclosure during infancy and childhood were misremembered and further shown how advocacy groups criticized former caregivers inappropriately. These accusations did little to enhance adjustment for the patient to improve future care. Patient's inaccurate memories from childhood events may result from confusing what parents said or from refusing to discuss unclear memories of stressful or traumatic experiences.

Patient no. 1 was diagnosed with 21-hydroxylase deficient $\mathrm{CAH}$ in the neonatal period and had feminizing genital surgery as an infant. She was compliant with medical therapy and had normal growth and pubertal development. Her medical records show that from late childhood, her routine visits included a discussion of $\mathrm{CAH}$, its diagnosis, therapy, consequences, and outcome. She required vaginoplasty while a late teenager to permit sexual intercourse. As a late teenager, she realized for the first time the impact of her condition, including the medical and surgical therapy, on her sexuality because of the diminished genital sensitivity and the risk of subfertility. Using a support group lawyer, this patient accused her primary CAH caregiver, a pediatric endocrinologist, of having never explained her condition and its potential consequences to her. The contact person in the support group was very careful not to accuse the endocrinologist, while the patient appeared to blame the potential consequences of her underlying condition on her endocrinologist. An attempt was made to give the patient a perspective that there is no perfect therapy for $\mathrm{CAH}$, and her physician had tried to make her aware of the possible consequences.

Patient \#2 has a diagnosis of 46,XY DSD and was born during the time period when the female gender assignment was commonly recommended for those with an unclear etiologic diagnosis and a phallus judged to be inadequate for intercourse. She was given a female sex assignment, and surgery was done in infancy to align with a female sex of rearing. The patient's mother indicated that she felt forced into the assignment, and in fact the medical record does not contain documentation of parental agreement with this decision. Throughout childhood, it was apparent that the mother, who accompanied the patient during clinic visits, had not fully accepted the female sex of rearing. Counseling was recommended and accepted by both the patient and the mother. However, the situation became worse during the teen years. Many of her problems appeared to stem from the situation in which her mother confided in other local mothers information about her daughter having been born with a very small penis, and so she was changed to a girl. As a consequence, her school peers of both sexes learned this information and teased her mercilessly. Faced with a degree of distress with which she did not know how to cope, this person has repeatedly accused her long-time caregiver, who assumed her medical care at age 6 years, of harmful medical treatment. In spite of providing this individual with medical records, accusations have continued that have been supported by her advocacy group. Such an unsatisfactory situation would hopefully be an opportunity for a support group to counsel and care for this patient to help her develop a perspective that her previous physicians had done what seemed best for her at the time. Unfortunately, she remains poorly adjusted, in need of helpful counseling having alienated a caregiver whose only intention was to help her.

Patient no. 3, is now in her 40s, was a $46, \mathrm{XX}$ patient who was initially assigned a male sex of rearing because of masculinized genitalia. At one month of age, after the diagnosis of $\mathrm{CAH}$ was made, she was reassigned female. At several years of age, care was transferred to a university affiliated hospital having a large pediatric endocrine unit. One endocrinologist subsequently assumed her primary endocrine care at age 14 years. At this time, she was a very attractive female, with normal weight and height for age, who was having regular menses. In fact, she was told how well she was doing. Subsequently she has said that she did not understand, if she was doing so well, why she had to continue to have regular doctor visits. She was unwilling or unable to accept the fact that she had a condition which would require lifelong monitoring and therapy adjustments. It became obvious while this patient was a teen that she had not adjusted well and that counseling was needed and began. She married and had severe sexual problems. She subsequently indicated to her previous caregiver that she had numerous problems because of $\mathrm{CAH}$ and its therapy, the primary one being sexual. Again counseling was recommended. More recently an advocacy group, on her behalf, initially contacted the hospital where she was cared for from early childhood through adolescence. The communication, consisting of a letter and regular-emails, demanded an apology for her previous care as a way of opening a dialogue to foster healing. These communications were forwarded to the hospital's legal department, where, after repeated demands for an 
apology, it was decided that a letter of apology be sent to recognize that harm may have occurred as a result of her then standard of care treatments. This letter was sent, even though the primary complaints were against the care during early infancy, before care was transferred to this hospital. Shortly thereafter, a similar legal advocacy letter was written to the physician who provided her pediatric endocrine care after age 14 years also demanding an apology for her care including nondisclosure, excessive examination room exposure to training physicians, and excessive genital examinations. In fact, these practices had changed prior to this physician assuming her care. In response to continued requests, a letter of apology was written even though legal advice indicated that there was no legal reason to do so and in spite of the perspective that it would do little to foster psychological adjustment. The letter was accompanied with an offer by the physician to discuss these issues directly with the patient. Subsequent to these two letters, a similar letter was written to the hospital where this person was initially treated as an infant. It would be hoped that with skillful counseling that this patient could have been spared the on-going psychological pain, with the former caregiver being part of the solution rather than the scapegoat of the problems.

\section{A Constructive Approach}

The care of individuals with CAH and other DSDs has evolved dramatically over the last two decades [16] and will likely evolve further as the team approach improves and refines management of those with such complicated and rare disorders. The changes have resulted from the realization of previous misconceptions about the determinants of gender identity, the assumption that sex of rearing can always override other determinants of gender identity, an appreciation of the impact of fetal androgen exposure, the recognition of the importance of adult female sexual responsiveness, and new methodologies to improve fertility potential.

This communication is a global plea for open and constructive communication among all members of the care team: the patient, the family, the practitioner and the support/advocacy group in the singular goal of improving patient outcomes. Not only should the traditional members of the multidisciplinary team caring for individuals with $\mathrm{CAH}$ and DSDs faithfully follow the Rotarian four-way test, but we, the authors, would submit that the newer members of the global approach, the support/advocacy groups, should also see this as a charter intended to improve patient outcomes, a goal upon which we can all agree.

\section{References}

[1] "Manual of Procedure, A Reference Manual for Rotary Leaders," 2007.

[2] J. Russell, "Can you survive Rotary's four-way test?" Journal Manage Engineering, vol. 16, article 13, 2000.

[3] S. Z. Miller and H. J. Schmidt, "The habit of humanism: a framework for making humanistic care a reflexive clinical skill," Academic Medicine, vol. 74, no. 7, pp. 800-803, 1999.
[4] P. F. Weissmann, W. T. Branch, C. F. Gracey, P. Haidet, and R. M. Frankel, "Role modeling humanistic behavior: learning bedside manner from the experts," Academic Medicine, vol. 81, no. 7, pp. 661-667, 2006.

[5] J. M. Wiecha and J. F. Markuns, "Promoting medical humanism: design and evaluation of an online curriculum," Family Medicine, vol. 40, no. 9, pp. 617-619, 2008.

[6] M. Diamond and H. K. Sigmundson, "Management of intersexuality. Guidelines for dealing with persons with ambiguous genitalia," Archives of Pediatrics \& Adolescent Medicine, vol. 151, no. 10, pp. 1046-1050, 1997.

[7] J. D. Bremner, K. K. Snobe, and J. F. Kihlstrom, "False memories in women with self-reported childhood sexual abuse: an empirical study," Psychological Science, vol. 11, no. 4, pp. 333-337, 2000.

[8] J. D. Bremner, E. Vermetten, N. Afzal, and M. Vythilingam, "Deficits in verbal declarative memory function in women with childhood sexual abuse-related posttraumatic stress disorder," Journal of Nervous and Mental Disease, vol. 192, no. 10, pp. 643-649, 2004.

[9] "CARES Foundation newsletter: preventing female virilization in congenital adrenal hyperplasia," vol. 9, Winter 2009.

[10] W. Ellen, M.D. Seely, A. David, M. D. Ehrmann, et al., Eds., "Preventing female virilization in congenital adrenal hyperplasia Female prenatal treatment of classic $\mathrm{CAH}$ with dexamethasome: Pro vs. Con From: The Endocrine Society's Research Affairs Core Committee," http://www.endosociety.org/endo_news/tri_point_series.cfm/.

[11] http://www.fetaldex.org/.

[12] L. Soriano-Guillén, N. Lahlou, G. Chauvet, M. Roger, J. L. Chaussain, and J. C. Carel, "Adult height after ketoconazole treatment in patients with familial male-limited precocious puberty," Journal of Clinical Endocrinology and Metabolism, vol. 90, no. 1, pp. 147-151, 2005.

[13] A. Tamar-Mattis, "Exploring gray areas in the law about DSD and sterilization," Endocrine Today, October 2009.

[14] A. Tamar-Mattis, "Giving pediatric patients a voice in treatment of DSD," Endocrine Today, March 2010.

[15] A. Tamar-Mattis, "Exploring gray areas in the law about DSD and sterilization," Endocrine Today. In press.

[16] P. A. Lee, C. P. Houk, S. F. Ahmed et al., "Consensus statement on management of intersex disorders," Pediatrics, vol. 118, no. 2, pp. e488-e500, 2006. 